This item was submitted to Loughborough's Research Repository by the author.

Items in Figshare are protected by copyright, with all rights reserved, unless otherwise indicated.

\title{
Infinite hierarchies of exact solutions of the Einstein and Einstein-Maxwell equations for interacting waves and inhomogeneous cosmologies
}

PLEASE CITE THE PUBLISHED VERSION

LICENCE

CC BY-NC-ND 4.0

\section{REPOSITORY RECORD}

Alekseev, G.A., and J.B. Griffiths. 2019. "Infinite Hierarchies of Exact Solutions of the Einstein and Einsteinmaxwell Equations for Interacting Waves and Inhomogeneous Cosmologies". figshare. https://hdl.handle.net/2134/748. 


\title{
Infinite hierarchies of exact solutions of the Einstein and Einstein-Maxwell equations for interacting waves and inhomogeneous cosmologies
}

\author{
G. A. Alekseev* \\ Steklov Mathematical Institute, \\ Gubkina 8, Moscow 117966, GSP-1, Moscow, Russia. \\ and J. B. Griffiths ${ }^{\dagger}$ \\ Department of Mathematical Sciences, Loughborough University \\ Loughborough, Leics. LE11 3TU, U.K.
}

April 12, 2000

\begin{abstract}
For space-times with two spacelike isometries, we present infinite hierarchies of exact solutions of the Einstein equations for vacuum and the Einstein-Maxwell equations for electrovacuum fields as represented by their Ernst potentials. These solutions are parametrized by three arbitrary rational functions of an auxiliary complex parameter and, therefore, depend on an arbitrarily large number of free parameters. They are constructed using a linear singular integral equation. This arises in the context of the so called "monodromy transform" approach, and is equivalent to the usual partial derivative form of space-time symmetry reduced Einstein equations. The solutions presented can be used to describe various inhomogeneous cosmological models, or gravitational and electromagnetic waves and their interactions. They include a number of important known solutions as particular cases.
\end{abstract}

\section{Introduction}

Infinite "hierarchies" of exact solutions of the Einstein and Einstein-Maxwell equations for vacuum and electrovacuum fields respectively are presented. They describe various configurations of nonlinearly interacting gravitational or gravitational and electromagnetic waves, or the evolution of some inhomogeneous vacuum or electrovacuum cosmological models. The solutions depend on two space-time coordinates only, but include an arbitrarily large number of free parameters. Varying these permits a wide range in the physical properties of the corresponding field configurations. They determine, for example, the profiles and polarizations of interacting waves, or the structure of cosmological models.

The known solution-generating techniques are based on the integrability properties of Einstein's equations for space-times with symmetries (viz. on the integrability of the Ernst equations). They were primarily designed to construct exact stationary axisymmetric solutions (with a trivial generalization for cylindrical waves) in which an additional regularity condition should be satisfied on the axis. However, this condition does not apply to interacting waves or cosmological models as considered here.

${ }^{*}$ Email address: G.A.Alekseev@mi.ras.ru

${ }^{\dagger}$ E-mail: J.B.Griffiths@Lboro.ac.uk 
Apart from the completely linearizable subcase of Einstein-Rosen vacuum gravitational waves, the only techniques which provide nontrivial tools for the construction of solutions for the dynamical case are the vacuum Belinskii-Zakharov inverse-scattering method [1], the so called "monodromy transform" approach $[2,3,4]$, and the group-theoretical approach recently developed by Hauser and Ernst [5]. In particular, the methods of [1] enable the construction of soliton perturbations of homogeneous cosmological models and some specific solutions for wave interaction regions. For example, the Khan-Penrose [7] or Nutku-Halil [8] solutions for the interaction region for colliding impulsive gravitational waves on a Minkowski background formally turn out to be two-soliton solutions on a symmetric Kasner background.

Here we consider the monodromy transform approach and the linear singular integral equations which arise in this context as an alternative form of the reduced Einstein equations. We present a new method for the solution of these equations which gives rise to infinite hierarchies of exact solutions. Among many other solutions, these include the particular cases mentioned above together with other soliton solutions on the symmetric Kasner background and their non-soliton extensions.

\section{Integral equation form of reduced Einstein equations}

According to methods developed in $[2,3,4]$, any solution of the Ernst equations can be constructed from the solution of the linear singular integral equation

$$
\frac{1}{\pi i} \int_{L} \frac{[\lambda]_{\zeta}}{\zeta-\tau} \mathcal{H}(\tau, \zeta) \varphi(\xi, \eta, \zeta) d \zeta=-\mathbf{k}(\tau)
$$

considered here for the hyperbolic case only. The parameters $\xi, \eta$ are two real null spacetime coordinates, e.g. $(\xi, \eta)=(x+t, x-t)$. These coordinates span some local region in the neighbourhood of some initial regular space-time point $P_{0}: \xi=\xi_{0}, \eta=\eta_{0}$, in which local solutions of the reduced Einstein equations are considered.

The integration in (1) is performed along the path $L$ on the spectral plane $w$ which consists of two disconnected parts $L_{+}$and $L_{-}$. In the hyperbolic case, these are chosen for simplicity as the segments of the real axis in the $w$-plane, which join the points $w=\xi_{0}$ and $w=\xi$, and $w=\eta_{0}$ and $w=\eta$ respectively. (We choose $\xi_{0} \neq \eta_{0}$ and take $\xi$ and $\eta$ sufficiently close to $\xi_{0}$ and $\eta_{0}$ that the segments $L_{ \pm}$do not overlap.)

The integral in (1) splits into two, one of which possesses a singular kernel of Cauchy type and should be understood as a Cauchy principal value integral. The integration parameter $\zeta$ and a parameter $\tau$ span both of the contours $L_{+}$and $L_{-}$. Sometimes it will be convenient to introduce suffices: $\zeta_{+}, \tau_{+} \in L_{+}$and $\zeta_{-}, \tau_{-} \in L_{-}$.

In the integrand in (1), $[\lambda]_{\zeta}=\frac{1}{2}\left(\lambda_{\text {left }}-\lambda_{\text {right }}\right)$. This represents the jump on the contour, i.e. half of the difference between left and right limit values at the point $\zeta \in L_{+}$or $\zeta \in L_{-}$of some "standard" function $\lambda(\xi, \eta, w)$. This function is a product of two functions $\lambda(\xi, \eta, w)=$ $\lambda_{+}(\xi, w) \lambda_{-}(\eta, w)$ given by

$$
\lambda_{+}=\sqrt{\frac{w-\xi}{w-\xi_{0}}}, \quad \lambda_{-}=\sqrt{\frac{w-\eta}{w-\eta_{0}}},
$$

with the additional conditions $\left.\lambda_{+}\right|_{w=\infty}=\left.\lambda_{-}\right|_{w=\infty}=1$. Each of these functions is an analytic function on the whole spectral plane $w$ apart from the cut $L_{+}$or $L_{-}$respectively, whose endpoints are the branching points of the corresponding function.

In the equations (1), the three-dimensional complex vector function $\varphi(\xi, \eta, \zeta)$ is unknown, and the right hand side $\mathbf{k}(\tau)$ is a three-dimensional complex vector function of the spectral parameter which may be taken to be

$$
\mathbf{k}(w)=\{1, \mathbf{u}(w), \mathbf{v}(w)\},
$$


where $\mathbf{u}(w)$ and $\mathbf{v}(w)$ are arbitrary functions. The kernel of the integral in (1) is a scalar function $\mathcal{H}(\tau, \zeta)$ given by

$$
\begin{gathered}
\mathcal{H}(\tau, \zeta)=1+i\left(\zeta-\beta_{0}\right)\left(\mathbf{u}(\tau)-\mathbf{u}^{\dagger}(\zeta)\right)+\alpha_{0}^{2} \mathbf{u}(\tau) \mathbf{u}^{\dagger}(\zeta) \\
-4\left(\zeta-\xi_{0}\right)\left(\zeta-\eta_{0}\right) \mathbf{v}(\tau) \mathbf{v}^{\dagger}(\zeta)
\end{gathered}
$$

where the dagger denotes complex conjugation: e.g. $\mathbf{u}^{\dagger}(w) \equiv \overline{\mathbf{u}(\bar{w})}$. The additional constants in $(4)$ are $\alpha_{0}=\left(\xi_{0}-\eta_{0}\right) / 2$ and $\beta_{0}=\left(\xi_{0}+\eta_{0}\right) / 2$.

It is important to emphasize that the integral equations (1), and hence the functions $\mathbf{u}(w)$, $\mathbf{v}(w)$ and $\boldsymbol{\varphi}(\xi, \eta, w)$, only need to be evaluated on the two cuts $L_{+}$and $L_{-}$in the spectral plane. Thus all the above vector and scalar functions of the spectral parameter are actually determined by pairs of functions which represent their values on these contours. For convenience we shall denote the values of these functions on $L_{ \pm}$by the corresponding suffices:

$$
\{\mathbf{u}(w), \mathbf{v}(w)\}= \begin{cases}\left\{\mathbf{u}_{+}(w), \mathbf{v}_{+}(w)\right\}, & w \in L_{+} \\ \left\{\mathbf{u}_{-}(w), \mathbf{v}_{-}(w)\right\}, & w \in L_{-}\end{cases}
$$

Thus, in (1) written in a more explicit form, we actually have two unknown vector functions $\boldsymbol{\varphi}_{ \pm}$. For any of these suffixed functions we can use also an alternative definition, for example,

$$
\boldsymbol{\varphi}\left(\xi, \eta, \tau_{ \pm}\right) \equiv \boldsymbol{\varphi}_{ \pm}(\xi, \eta, \tau) .
$$

Using this notation, it is convenient to split the integral in (1) into separate integrals over $L_{+}$ and $L_{-}$and to consider separately the cases $\tau=\tau_{+} \in L_{+}$and $\tau=\tau_{-} \in L_{-}$. It is also convenient to denote the four scalar kernels which appear in the integrands of (1) in the form

$$
\begin{aligned}
\mathcal{H}\left(\tau_{+}, \zeta_{+}\right) & \equiv \mathcal{H}_{++}(\tau, \zeta), & & \mathcal{H}\left(\tau_{+}, \zeta_{-}\right) \equiv \mathcal{H}_{+-}(\tau, \zeta), \\
\mathcal{H}\left(\tau_{-}, \zeta_{+}\right) & \equiv \mathcal{H}_{-+}(\tau, \zeta), & & \mathcal{H}\left(\tau_{-}, \zeta_{-}\right) \equiv \mathcal{H}_{--}(\tau, \zeta)
\end{aligned}
$$

where the functions $\mathcal{H}_{++}(\tau, \zeta), \mathcal{H}_{+-}(\tau, \zeta), \mathcal{H}_{-+}(\tau, \zeta)$ and $\mathcal{H}_{--}(\tau, \zeta)$ can be determined explicitly in terms of the four functions $\mathbf{u}_{ \pm}(w)$ and $\mathbf{v}_{ \pm}(w)$ using (4).

To conclude our description of the structure of the master integral equations, we recall that the four functions $\mathbf{u}_{ \pm}(w)$ and $\mathbf{v}_{ \pm}(w)$ appearing in (3) and (5) play a significant role in the entire construction. They determine completely the coefficients of the integral equations in the electrovacuum case. In the vacuum case there are only two such functions $\mathbf{u}_{ \pm}(w)$, as $\mathbf{v}_{ \pm}(w) \equiv 0$. As shown in [3], they characterize unambiguously every individual solution of the Ernst equations. Moreover, the singular integral equations (1) possess a unique solution for any given choice of analytical functions $\mathbf{u}_{ \pm}(w)$ and $\mathbf{v}_{ \pm}(w)$.

We recall now also, that the general local solution of the hyperbolic Ernst equations can be expressed by quadratures in terms of the solution of (1)

$$
\begin{aligned}
& \mathcal{E}=-1-\frac{2}{\pi} \int_{L}[\lambda]_{\zeta}\left[1-i\left(\zeta-\beta_{0}\right) \mathbf{u}^{\dagger}(\zeta)\right] \boldsymbol{\varphi}^{[u]}(\xi, \eta, \zeta) d \zeta \\
& \Phi=\frac{2}{\pi} \int_{L}[\lambda]_{\zeta}\left[1-i\left(\zeta-\beta_{0}\right) \mathbf{u}^{\dagger}(\zeta)\right] \boldsymbol{\varphi}^{[v]}(\xi, \eta, \zeta) d \zeta
\end{aligned}
$$

where $\varphi^{[u]}$ and $\varphi^{[v]}$, in some association with the definition (3), denote respectively the second and third components of the vector solutions $\boldsymbol{\varphi}$ of the master integral equation (1), corresponding to a given choice of the monodromy data functions $\mathbf{u}_{ \pm}(w)$ and $\mathbf{v}_{ \pm}(w)$. In a more explicit form, each of the the integrals in (6) should be split into two integrals evaluated over $L_{+}$and $L_{-}$.

\section{New hierarchies of solutions}

Here we will construct a class of hyperbolic solutions that is determined by the rational monodromy data

$$
\mathbf{u}_{ \pm}(w)=\frac{U_{ \pm}(w)}{Q_{ \pm}(w)}, \quad \mathbf{v}_{ \pm}(w)=\frac{V_{ \pm}(w)}{Q_{ \pm}(w)}
$$


where $U_{+}(w), V_{+}(w), Q_{+}(w)$ and $U_{-}(w), V_{-}(w), Q_{-}(w)$ are arbitrary complex polynomials, provided $\mathbf{u}_{+}(w), \mathbf{v}_{+}(w)$ and $\mathbf{u}_{-}(w), \mathbf{v}_{-}(w)$ do not have poles on $L_{+}$and $L_{-}$respectively.

For what follows, it is convenient to calculate some auxiliary polynomials of two variables we introduce the four polynomials $P_{ \pm \pm}(\tau, \zeta)$ defined by the relations

$$
\mathcal{H}_{ \pm \pm}(\tau, \zeta)=\frac{P_{ \pm \pm}(\tau, \zeta)}{Q_{ \pm}(\tau) Q_{ \pm}^{\dagger}(\zeta)}
$$

and four polynomials $R_{ \pm \pm}(\tau, \zeta)$ defined from them by

$$
R_{ \pm \pm}(\tau, \zeta)=\frac{P_{ \pm \pm}(\tau, \zeta)-P_{ \pm \pm}(\zeta, \zeta)}{\zeta-\tau} .
$$

In these definitions there are two sets of suffices, denoted as dotted and undotted, which should each be taken to be the same. Finally, it is convenient to introduce a redefinition of the unknown functions

$$
\begin{gathered}
\boldsymbol{\varphi}_{+}(\zeta)=-\frac{\lambda_{-}^{-1}(\zeta) Q_{+}^{\dagger}(\zeta)}{P_{++}(\zeta, \zeta)} \widetilde{\boldsymbol{\varphi}}_{+}(\zeta), \\
\boldsymbol{\varphi}_{-}(\zeta)=-\frac{\lambda_{+}^{-1}(\zeta) Q_{-}^{\dagger}(\zeta)}{P_{--}(\zeta, \zeta)} \widetilde{\boldsymbol{\varphi}}_{-}(\zeta) .
\end{gathered}
$$

Hereafter we do not show explicitly the arguments $\xi$ and $\eta$ of $\boldsymbol{\varphi}_{ \pm}$and $\lambda$ or the suffices \pm at the points $\zeta$ and $\tau$, unless it is necessary.

A direct substitution of (7) into equations (1) with the use of (8)-(10) leads to the following convenient form of linear equations with polynomial right hand sides

$$
\begin{aligned}
\frac{1}{\pi i} \int_{\xi_{0}}^{\xi} \frac{\left[\lambda_{+}\right]_{\zeta}}{\zeta-\tau_{+}} \widetilde{\boldsymbol{\varphi}}_{+}(\zeta) d \zeta=-\frac{1}{\pi i} \int_{\xi_{0}}^{\xi}\left[\lambda_{+}\right]_{\zeta} \frac{R_{++}\left(\tau_{+}, \zeta\right)}{P_{++}(\zeta, \zeta)} \widetilde{\boldsymbol{\varphi}}_{+}(\zeta) d \zeta & -\frac{1}{\pi i} \int_{\eta_{0}}^{\eta}\left[\lambda_{-}\right]_{\zeta} \frac{R_{+-}\left(\tau_{+}, \zeta\right)}{P_{--}(\zeta, \zeta)} \widetilde{\boldsymbol{\varphi}}_{-}(\zeta) d \zeta+\left(\begin{array}{c}
Q_{+}\left(\tau_{+}\right) \\
U_{+}\left(\tau_{+}\right) \\
V_{+}\left(\tau_{+}\right)
\end{array}\right), \\
\frac{1}{\pi i} \int_{\eta_{0}}^{\eta} \frac{\left[\lambda_{-}\right]_{\zeta}}{\zeta-\tau_{-}} \widetilde{\boldsymbol{\varphi}}_{-}(\zeta) d \zeta=-\frac{1}{\pi i} \int_{\eta_{0}}^{\eta}\left[\lambda_{-}\right]_{\zeta} \frac{R_{--}\left(\tau_{-}, \zeta\right)}{P_{--}(\zeta, \zeta)} \widetilde{\boldsymbol{\varphi}}_{-}(\zeta) d \zeta & -\frac{1}{\pi i} \int_{\xi_{0}}^{\xi}\left[\lambda_{+}\right]_{\zeta} \frac{R_{-+}\left(\tau_{-}, \zeta\right)}{P_{++}(\zeta, \zeta)} \widetilde{\boldsymbol{\varphi}}_{+}(\zeta) d \zeta+\left(\begin{array}{c}
Q_{-}\left(\tau_{-}\right) \\
U_{-}\left(\tau_{-}\right) \\
V_{-}\left(\tau_{-}\right)
\end{array}\right)
\end{aligned}
$$

if we impose constraints on the coefficients of the rational functions (7) such that

$$
P_{+-}(\zeta, \zeta)=P_{-+}(\zeta, \zeta)=0 .
$$

This leads to a large class of explicit solutions $\widetilde{\boldsymbol{\varphi}}_{ \pm}(\xi, \eta, \tau)$ of $(11)$ that are regular on the cuts $L_{ \pm}$. However, the solution of the Ernst equations needs the solutions $\boldsymbol{\varphi}_{ \pm}(\xi, \eta, \tau)$ of (1) to be regular on the cuts $L_{ \pm}$. Fortunately, all additional singularities (poles) of $\boldsymbol{\varphi}_{+}(\xi, \eta, \tau)$ on $L_{+}$and $\boldsymbol{\varphi}_{-}(\xi, \eta, \tau)$ on $L_{-}$, which arise from the denominators in (10), can be avoided by the additional restrictions that $\mathbf{u}_{+}\left(\eta_{0}\right)=-i / \alpha_{0}$ and $\mathbf{u}_{-}\left(\xi_{0}\right)=i / \alpha_{0}$. We therefore specify

$$
\begin{aligned}
& \mathbf{u}_{+}(w)=-\frac{i}{\alpha_{0}}+\left(w-\eta_{0}\right) \frac{C_{+}(w)}{Q_{+}(w)} \\
& \mathbf{u}_{-}(w)=\frac{i}{\alpha_{0}}+\left(w-\xi_{0}\right) \frac{C_{-}(w)}{Q_{-}(w)}
\end{aligned}
$$


where $C_{+}(w), C_{-}(w), Q_{+}(w)$ and $Q_{-}(w)$ are arbitrary polynomials. With these, the ansatz (12) leads to the constraint $C_{-}(w)=B(w) C_{+}^{\dagger}(w)-4 i A(w) V_{+}^{\dagger}(w)$ and, for the polynomials in (7), the general solution of (12) reads

$$
\begin{aligned}
& U_{+}(w)=-\frac{i}{\alpha_{0}} Q_{+}(w)+\left(w-\eta_{0}\right) C_{+}(w) \\
& U_{-}(w)=B(w)\left(\frac{i}{\alpha_{0}} Q_{+}(w)+\left(w-\beta_{0}\right) C_{+}^{\dagger}(w)\right)-4 i\left(w-\xi_{0}\right) A(w) V_{+}^{\dagger}(w) \\
& V_{-}(w)=A(w)\left(Q_{+}^{\dagger}(w)-i \alpha_{0}^{2} C_{+}^{\dagger}(w)\right) \\
& Q_{-}(w)=B(w)\left(Q_{+}^{\dagger}(w)-i \alpha_{0}^{2} C_{+}^{\dagger}(w)\right)
\end{aligned}
$$

where the polynomials $A(w), B(w), C_{+}(w), V_{+}(w)$ and $Q_{+}(w)$ can be chosen arbitrarily, provided the corresponding functions $\mathbf{u}_{ \pm}(w), \mathbf{v}_{ \pm}(w)$ have no poles on the cuts $L_{+}$and $L_{-}$respectively. The vacuum case, which occurs when $A(w)=V_{+}(w)=0$ and $B(w)=1$, yields simpler expressions which involve just two arbitrary polynomials $C_{+}(w)$ and $Q_{+}(w)$.

Returning to (11), we note that the integral operators in the left hand sides can be inverted using the Poincaré-Bertrand formula [6] for singular integrals

$$
\frac{1}{\pi i} \int_{L} \frac{[\lambda]_{\zeta}}{\zeta-\tau} \boldsymbol{\varphi}(\zeta) d \zeta=f(\tau) \quad \Leftrightarrow \quad \boldsymbol{\varphi}(\tau)=\frac{1}{\pi i} \int_{L} \frac{\left[\lambda^{-1}\right]_{\zeta}}{\zeta-\tau} f(\zeta) d \zeta
$$

This can be applied to the integrals over $L_{+}$(using $\lambda_{+}$), or over $L_{-}$(using $\lambda_{-}$).

Since the right hand sides of (11) are polynomials in $\tau$, the inversion (15) leads to the solution in the form

$$
\widetilde{\boldsymbol{\varphi}}_{ \pm}(\tau)=\sum_{k=0}^{N_{ \pm}}\left(\begin{array}{c}
\widetilde{q}_{k_{ \pm}} \\
\widetilde{u}_{k_{ \pm}} \\
\widetilde{v}_{k_{ \pm}}
\end{array}\right) Z_{k \pm}(\tau)
$$

where $N_{+}$and $N_{-}$are the maxima of the degrees of the polynomials $U_{+}, V_{+}, Q_{+}$and $U_{-}, V_{-}$, $Q_{-}$respectively, $\widetilde{u}_{k \pm}, \widetilde{v}_{k \pm}, \widetilde{q}_{k \pm}$ are unknown $\tau$-independent functions of $\xi$ and $\eta$, and $Z_{k \pm}(\tau)$ are "standard" functions given by

$$
Z_{k \pm}(\tau)=\frac{1}{\pi i} \int_{L_{ \pm}} \frac{\left[\lambda_{ \pm}^{-1}\right]_{\zeta}}{\zeta-\tau} \zeta^{k} d \zeta
$$

All these functions (integrals) can be evaluated as the residues of their integrands at $\zeta=\infty$ are polynomials in $\tau$ of degree $k$.

We note now, that the vector integral equations (1) decouple into three pairs of equations - one pair for each of the three components of $\widetilde{\boldsymbol{\varphi}}_{+}$and the corresponding component of $\widetilde{\boldsymbol{\varphi}}_{-}$. All these pairs of equations possess the same kernels but different right hand sides. Therefore, substituting the expressions (16) into (11) and using (9) with (12), we get three decoupled algebraic systems, each of order $(N+2) \times(N+2)$ where $N=N_{+}+N_{-}$and for the sets of unknowns $\widetilde{q}_{k \pm}, \widetilde{u}_{k \pm}, \widetilde{v}_{k \pm}$ respectively. However, in view of (6), we need the solutions of two of these systems only:

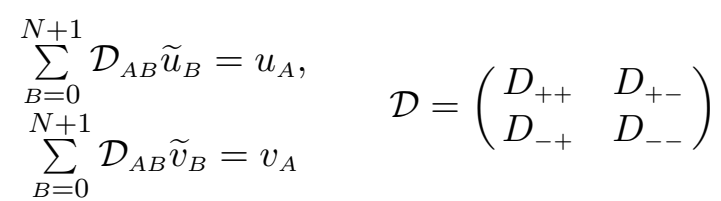

where the indices $A, B=0,1, \ldots N+1$. The column vectors $u_{A}, v_{A}$ (shown below as rows) are composed of the coefficients of the polynomials $U_{ \pm}(\zeta)$ and $V_{ \pm}(\zeta)$ :

$$
\begin{aligned}
& u_{A}=\left\{u_{0+}, u_{1+}, \ldots, u_{N_{+}}, u_{0_{-}}, u_{1_{-}}, \ldots, u_{N_{-}}\right\} \\
& v_{A}=\left\{v_{0+}, v_{1+}, \ldots, v_{N_{+}}, v_{0_{-}}, v_{1_{-}}, \ldots, v_{N_{-}}\right\} .
\end{aligned}
$$


Similarly, we combine the coefficients $\widetilde{u}_{k \pm}, \widetilde{v}_{k \pm}$ in (16) to form the column vectors (rows)

$$
\begin{aligned}
& \widetilde{u}_{A}(\xi, \eta)=\left\{\widetilde{u}_{0+}, \widetilde{u}_{1+}, \ldots, \widetilde{u}_{N_{+}}, \widetilde{u}_{0_{-}}, \widetilde{u}_{1_{-}}, \ldots, \widetilde{u}_{N_{-}}\right\} \\
& \widetilde{v}_{A}(\xi, \eta)=\left\{\widetilde{v}_{0_{+}}, \widetilde{v}_{1+}, \ldots, \widetilde{v}_{N_{+}}, \widetilde{v}_{0_{-}}, \widetilde{v}_{1_{-}}, \ldots, \widetilde{v}_{N_{-}}\right\}
\end{aligned}
$$

The matrix $\|\mathcal{D}\|$ consists of the blocks $D_{++}, D_{+-}, D_{-+}, D_{--}$of orders $\left(N_{+}+1\right) \times\left(N_{+}+1\right)$, $\left(N_{+}+1\right) \times\left(N_{-}+1\right),\left(N_{-}+1\right) \times\left(N_{+}+1\right)$ and $\left(N_{-}+1\right) \times\left(N_{-}+1\right)$ respectively. Their components are determined by the integrals:

$$
\begin{aligned}
& \left(D_{++}\right)_{k l}(\xi)=\delta_{k l}+\frac{1}{\pi i} \int_{\xi_{0}}^{\xi}\left[\lambda_{+}\right]_{\zeta} \frac{\left(R_{++}\right)_{k}(\zeta)}{P_{++}(\zeta, \zeta)} Z_{l+}(\zeta) d \zeta \\
& \left(D_{+-}\right)_{k l}(\eta)=\frac{1}{\pi i} \int_{\eta_{0}}^{\eta}\left[\lambda_{-}\right]_{\zeta} \frac{\left(R_{+-}\right)_{k}(\zeta)}{P_{--}(\zeta, \zeta)} Z_{l-}(\zeta) d \zeta \\
& \left(D_{-+}\right)_{k l}(\xi)=\frac{1}{\pi i} \int_{\xi_{0}}^{\xi}\left[\lambda_{+}\right]_{\zeta} \frac{\left(R_{-+}\right)_{k}(\zeta)}{P_{++}(\zeta, \zeta)} Z_{l+}(\zeta) d \zeta \\
& \left(D_{--}\right)_{k l}(\eta)=\delta_{k l}+\frac{1}{\pi i} \int_{\eta_{0}}^{\eta}\left[\lambda_{-}\right]_{\zeta} \frac{\left(R_{--}\right)_{k}(\zeta)}{P_{--}(\zeta, \zeta)} Z_{l-}(\zeta) d \zeta
\end{aligned}
$$

where $\left(R_{ \pm \pm}\right)_{k}$ are the coefficients in the expansions $R_{+ \pm}(\tau, \zeta)=\sum_{k=0}^{N_{+}}\left(R_{+ \pm}\right){ }_{k}(\zeta) \tau^{k}$ and $R_{- \pm}(\tau, \zeta)=$ $\sum_{k=0}^{N_{-}}\left(R_{- \pm}\right)_{k}(\zeta) \tau^{k}$

To calculate the final expressions for the Ernst potentials, we need to evaluate the additional sets of integrals

$$
\begin{gathered}
J_{k+}(\xi)=\frac{1}{\pi i} \int_{\xi_{0}}^{\xi}\left[\lambda_{+}\right]_{\zeta} \frac{Q_{+}^{\dagger}(\zeta)-i\left(\zeta-\beta_{0}\right) U_{+}^{\dagger}(\zeta)}{P_{++}(\zeta, \zeta)} Z_{k+}(\zeta) d \zeta \\
J_{k-}(\eta)=\frac{1}{\pi i} \int_{\eta_{0}}^{\eta}\left[\lambda_{-}\right]_{\zeta} \frac{Q_{-}^{\dagger}(\zeta)-i\left(\zeta-\beta_{0}\right) U_{-}^{\dagger}(\zeta)}{P_{--}(\zeta, \zeta)} Z_{k-}(\zeta) d \zeta,
\end{gathered}
$$

and to combine them into one row vector

$$
J_{A}=\left\{J_{0+}, J_{1+}, \ldots, J_{N_{+}}, J_{0_{-}}, J_{1-}, \ldots, J_{N_{-}}\right\} .
$$

Let us also define two additional $(N+2) \times(N+2)$ matrices

$$
\mathcal{G}_{A B}=\mathcal{D}_{A B}-2 i u_{A} J_{B}, \quad \mathcal{F}_{A B}=\mathcal{D}_{A B}-2 i v_{A} J_{B} .
$$

All integrals determining the components of the matrices $\mathcal{G}_{A B}, \mathcal{F}_{A B}$ and $\mathcal{D}_{A B}$ can be evaluated in terms of the residues of their integrands at the zeros of $P_{++}(w, w)$ and $P_{--}(w, w)$ and at $w=\infty$. We then have

$$
\mathcal{E}=-\frac{\operatorname{det}\left\|\mathcal{G}_{A B}\right\|}{\operatorname{det}\left\|\mathcal{D}_{A B}\right\|}, \quad \Phi=\frac{\operatorname{det}\left\|\mathcal{F}_{A B}\right\|}{\operatorname{det}\left\|\mathcal{D}_{A B}\right\|},
$$

which are the final expressions for the Ernst potentials. These solutions generally possess essentially nonlinear properties. They are not trivial time-dependent analogues of any stationary axisymmetric solutions with regular axis of symmetry which have different structures of monodromy data. The expressions (24) generally are not rational functions of $\xi, \eta$.

When evaluating explicit examples, it may be noted that solutions with a diagonal metric occur when $\mathbf{u}_{ \pm}^{\dagger}=-\mathbf{u}_{ \pm}$. The plane symmetric (type D) Kasner metric with $\mathcal{E}=-\alpha / \alpha_{0}$ is 
obtained using the constants $\mathbf{u}_{+}=-i / \alpha_{0}, \mathbf{u}_{-}=i / \alpha_{0}$ and $\mathbf{v}_{ \pm}=0$. The Khan-Penrose solution [7] for colliding plane impulsive gravitational waves is obtained with $\mathbf{v}_{+}(w)=\mathbf{v}_{-}(w)=0$ and

$$
\mathbf{u}_{+}(w)=i k_{+} \frac{w-a_{+}}{w-b_{+}}, \quad \mathbf{u}_{-}(w)=i k_{-} \frac{w-a_{-}}{w-b_{-}}
$$

when the constants $a_{ \pm}, b_{ \pm}$and $k_{ \pm}$are real. The nondiagonal Nutku-Halil solution [8] for noncolinear impulsive waves is obtained from the same expression using complex constants. This explicitly demonstrates that the above method is applicable to both the linear and nonlinear cases.

This work was partly supported by the EPSRC and by the grants 99-01-01150 and 99-0218415 from the RFBR.

\section{References}

[1] V. A. Belinskii and V. E. Zakharov, Sov. Phys. JETP 48 (1978) 985

[2] G. A. Alekseev, Dokl. Akad. Nauk SSSR, 283, 577 (1985); Sov. Phys. Dokl. 30, 565 (1985).

[3] G. A. Alekseev, Tr. Mat. Inst. Steklova, 176, 211 (1987); Proc. Steklov Inst. Maths. 3, 215 (1988).

[4] G. A. Alekseev, gr-qc/9911045, gr-qc/9912109.

[5] I. Hauser and F. J. Ernst, gr-qc/9903104, gr-qc/0002049.

[6] F. D. Gakhov, Boundary value problems, 3rd Russian ed. "Nauka", Moscow (1977): English translation of 2nd ed. Pergamon Press, Oxford (1966).

[7] K. A. Khan and R. Penrose, Nature, 229, 185 (1971).

[8] Y. Nutku and M. Halil, Phys. Rev. Lett., 39, 1379 (1977). 